\title{
Modeling photonastic materials: a first computational study.
}

\author{
Laura Le Bras,$^{\dagger}$ Claire Lemarchand, ${ }^{\ddagger}$ Stéphane Aloïse, ${ }^{\S}$ Carlo Adamo, ${ }^{\dagger}, \|$ \\ Nicolas Pineau, $*, \uparrow, \uparrow$ and Aurélie Perrier ${ }^{*, \dagger, \perp}$ \\ $\dagger$ Chimie ParisTech, PSL Research University, CNRS, Institute of Chemistry for Life and \\ Health Sciences (i-CLeHS), F-75005 Paris, France \\ $\ddagger C E A / D A M / D I F, 91297$ Arpajon Cedex, France \\ ๆUniversité Paris-Saclay, CEA, Laboratoire Matière sous Conditions Extrêmes, 91680, \\ Bruyères-le-Chatel, France. \\ §Univ. Lille, CNRS, UMR 8516 - LASIR - Laboratoire de Spectrochimie Infrarouge et \\ Raman, F-59000 Lille, France. \\ || Institut Universitaire de France, 103 Bd Saint-Michel, F-75005 Paris - France \\ $\perp$ Université de Paris, F-75006 Paris, France \\ E-mail: n.pineau@cea.fr; aurelie.perrier-pineau@u-paris.fr
}




\section{Validation of the GAFF implementation in the STAMP code.}

To validate the implementation of GAFF in the STAMP code, we have compared the structures of the OF-AP, OF-P and CF isomers of theDTE-Upy molecules, obtained after:

- a minimization process in vacuum with GAFF with the AMBER18 suite ${ }^{1}$

- a minimization process in vacuum with GAFF with STAMP

In both cases, periodic boundary conditions are not taken into account and the steepest descent algorithm is considered. In Table S1, some important structural parameters are compared.

Table S1: Selected geometrical parameters obtained after minimization for the isomers OFAP, OF-P and CF isomers with GAFF implemented in STAMP and Amber18. The distances are in Angstrom, the dihedral angles are in degrees. The structural parameters are defined on Figure S1 and in Table S2.

\begin{tabular}{c|cccccc}
\hline & \multicolumn{2}{|c}{ OF-AP } & \multicolumn{2}{c}{ OF-P } & \multicolumn{2}{c}{ CF } \\
& STAMP & Amber18 & STAMP & Amber18 & STAMP & Amber18 \\
\hline Distances & & & & & & \\
$\mathrm{d}_{c c}$ & 3.44 & 3.44 & 4.18 & 4.12 & 1.54 & 1.57 \\
$\mathrm{~d}_{1}$ & 19.38 & 19.30 & 28.20 & 28.28 & 28.71 & 28.56 \\
$\mathrm{~d}_{2}$ & 23.15 & 23.07 & 26.67 & 26.56 & 24.94 & 24.87 \\
$\mathrm{~d}_{3}$ & 14.22 & 13.90 & 15.36 & 15.55 & 15.54 & 15.58 \\
$\mathrm{~d}_{4}$ & 14.12 & 14.29 & 15.46 & 15.69 & 15.48 & 15.58 \\
$\mathrm{~d}$ & 28.34 & 27.86 & 19.38 & 19.41 & 26.68 & 26.10 \\
Dihedral angles & & & & & & \\
$\Phi_{1}$ & -61 & -54 & 26 & 22 & -20 & -14 \\
$\Phi_{2}$ & -14 & -14 & -122 & -124 & 5 & 1 \\
\hline
\end{tabular}

For the three isomers, the difference between the structures obtained after a minimization with STAMP or Amber18 are trifling. Indeed, for the selected distances, slight deviations (¡2 \%) are observed between the structures minimized with GAFF with STAMP or the Amber18 code.

After the structural comparison, we also investigated the optical properties of the resulting structures (Table S3). As it was expected due to the structure similarity, the optical prop- 


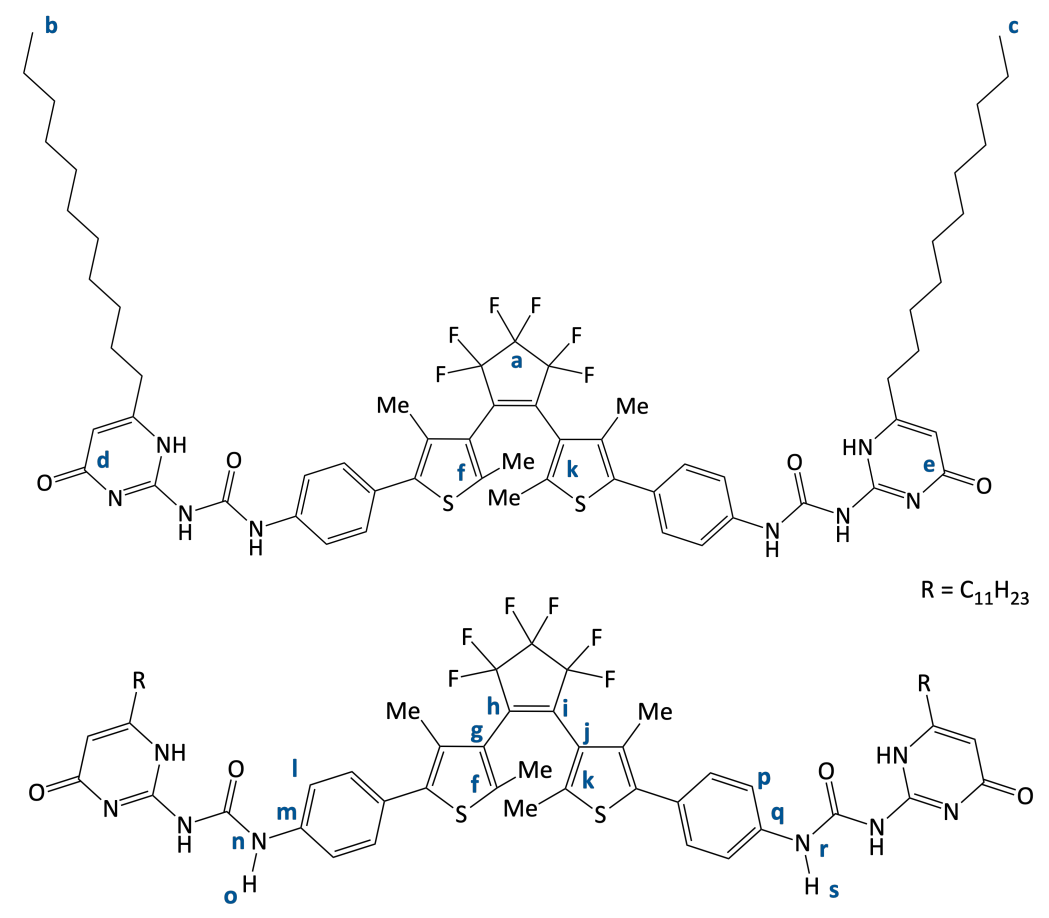

Figure S1: Definition of some key structural parameters: atom numbering.

Table S2: Definition of some key structural parameters: key distances and dihedral angles.

\begin{tabular}{llll}
\hline Distances & & Dihedrals & \\
$\mathrm{d}_{1}$ & $\mathrm{C}_{\mathrm{a}}-\mathrm{C}_{\mathrm{b}}$ & $\phi_{1}$ & $\mathrm{C}_{\mathrm{i}}-\mathrm{C}_{\mathrm{h}}-\mathrm{C}_{\mathrm{g}}-\mathrm{C}_{\mathrm{f}}$ \\
$\mathrm{d}_{2}$ & $\mathrm{C}_{\mathrm{a}}-\mathrm{C}_{\mathrm{c}}$ & $\phi_{2}$ & $\mathrm{C}_{\mathrm{h}}-\mathrm{C}_{\mathrm{i}}-\mathrm{C}_{\mathrm{j}}-\mathrm{C}_{\mathrm{k}}$ \\
$\mathrm{d}_{3}$ & $\mathrm{C}_{\mathrm{d}}-\mathrm{C}_{\mathrm{b}}$ & $\beta_{1}$ & $\mathrm{C}_{\mathrm{l}}-\mathrm{C}_{\mathrm{m}}-\mathrm{N}_{\mathrm{n}}-\mathrm{H}_{\mathrm{o}}$ \\
$\mathrm{d}_{4}$ & $\mathrm{C}_{\mathrm{e}}-\mathrm{C}_{c}$ & $\beta_{2}$ & $\mathrm{C}_{\mathrm{p}}-\mathrm{C}_{\mathrm{q}}-\mathrm{N}_{\mathrm{r}}-\mathrm{H}_{\mathrm{s}}$ \\
$\mathrm{d}^{\prime}$ & $\mathrm{C}_{\mathrm{d}}-\mathrm{C}_{\mathrm{e}}$ & \\
$\mathrm{d}_{\mathrm{cc}}$ & $\mathrm{C}_{\mathrm{f}}-\mathrm{C}_{\mathrm{k}}$ & \\
\hline
\end{tabular}

Table S3: Impact of the geometries on the maximum absorption wavelength $\left(\lambda_{\max }\right)$ calculated at the CAM-B3LYP/6-3111+G(2d,p) with Gaussian16 for the isomers OF-AP, OF-P and CF. Two different GAFF geometries are considered for each isomer: the one optimized with STAMP and the one optimized with Amber18.

\begin{tabular}{l|cccccc}
\hline & \multicolumn{2}{|c}{ OF-AP } & \multicolumn{2}{c}{ OF-P } & \multicolumn{2}{c}{ CF } \\
& STAMP & GAFF & GAFF@STAMP & GAFF & GAFF@STAMP & GAFF \\
\hline$\lambda_{\max }$ & 265 & 267 & 274 & 272 & 538 & 530 \\
\hline
\end{tabular}

erties are also similar for the structures obtained after a minimization process in STAMP or in Amber18. 


\section{Impact of the polymer matrix on the properties of DTE- Upy photochrom: complementary analysis of the MD simulations.}

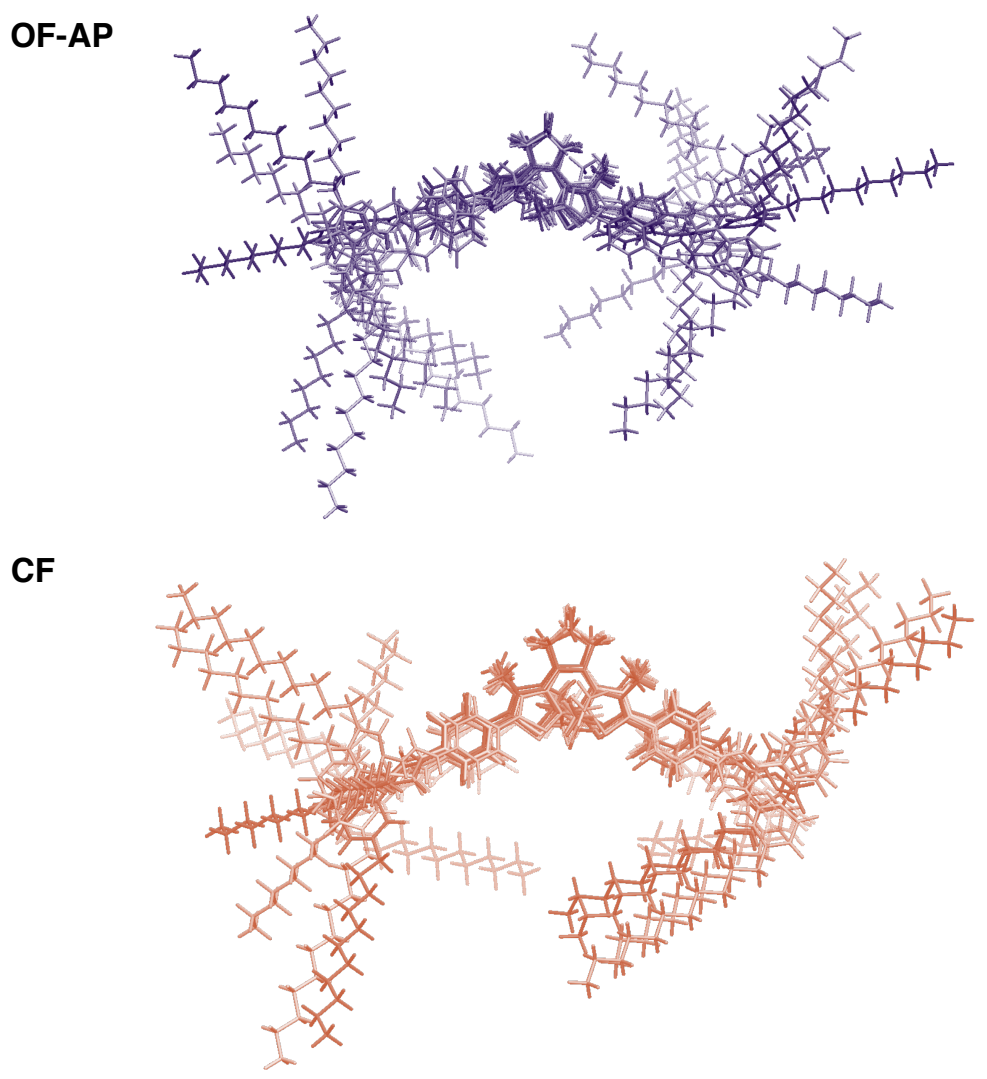

Figure S2: OF-AP and CF isomers: representation of the 10 structures obtained after the CPL process and used as an intial structure for the 10 simulations in the polybutadiene matrix (STAMP) and in the chroroform (Amber18). 
Table S4: Average values for different distances (in Angstroms, $\AA$ ) and dihedral angles (in degrees) for the nine others "1 ns" trajectories launched for the OF-AP conformer within the polymer matrix.

\begin{tabular}{l|cccccccc}
\hline & $\mathrm{d}_{c c}$ & $\mathrm{~d}_{1}$ & $\mathrm{~d}_{2}$ & $\mathrm{~d}_{3}$ & $\mathrm{~d}_{4}$ & $\mathrm{~d}^{\prime}$ & $\phi_{1}$ & $\phi_{2}$ \\
\hline $\mathbf{O F}_{\mathbf{A}-\mathbf{A P}_{2}}$ & 3.76 & 22.90 & 22.81 & 15.13 & 14.83 & 28.48 & -24 & -61 \\
$\mathbf{O F}-\mathbf{A P}_{3}$ & 3.78 & 26.13 & 20.44 & 15.24 & 14.59 & 26.09 & -23 & -64 \\
OF-AP $_{4}$ & 3.66 & 23.21 & 24.99 & 15.81 & 15.66 & 28.43 & -15 & -65 \\
OF-AP $_{5}$ & 3.71 & 24.04 & 14.81 & 13.58 & 16.08 & 29.31 & -30 & -57 \\
OF-AP $_{6}$ & 3.70 & 19.38 & 15.03 & 15.94 & 15.53 & 27.93 & -21 & -63 \\
OF-AP $_{7}$ & 3.79 & 19.02 & 17.73 & 15.82 & 16.82 & 26.69 & -26 & -59 \\
OF-AP $_{8}$ & 3.71 & 16.24 & 13.36 & 15.23 & 15.36 & 29.35 & -27 & -62 \\
OF-AP $_{9}$ & 3.77 & 29.90 & 24.24 & 15.89 & 14.08 & 27.04 & -17 & -51 \\
OF-AP $_{10}$ & 3.77 & 27.44 & 25.70 & 14.25 & 14.78 & 28.57 & -16 & -66 \\
\hline
\end{tabular}

Table S5: Average values for different distances (in Angstroms, $\AA$ ) and dihedral angles (in degrees) for the nine others 1 ns trajectories launched for the OF-P conformerwithin the polymer matrix.

\begin{tabular}{l|cccccccc}
\hline & $\mathrm{d}_{c c}$ & $\mathrm{~d}_{1}$ & $\mathrm{~d}_{2}$ & $\mathrm{~d}_{3}$ & $\mathrm{~d}_{4}$ & $\mathrm{~d}^{\prime}$ & $\phi_{1}$ & $\phi_{2}$ \\
\hline $\mathbf{O F}_{2}$ & 4.07 & 18.15 & 19.68 & 13.16 & 13.03 & 22.99 & 20 & -120 \\
OF-P $_{3}$ & 4.10 & 21.84 & 23.13 & 12.06 & 13.35 & 21.72 & 20 & -123 \\
OF-P $_{4}$ & 4.12 & 22.64 & 21.66 & 14.09 & 13.21 & 22.00 & 20 & -123 \\
OF-P $_{5}$ & 4.01 & 22.92 & 21.68 & 12.49 & 14.19 & 22.21 & 20 & -123 \\
OF-P $_{6}$ & 4.07 & 21.20 & 18.53 & 13.96 & 12.96 & 20.30 & 22 & -125 \\
OF-P $_{7}$ & 4.09 & 17.06 & 18.67 & 12.37 & 12.94 & 21.17 & 24 & -121 \\
OF-P $_{8}$ & 4.09 & 22.08 & 19.91 & 14.60 & 13.66 & 20.66 & 21 & -124 \\
OF-P $_{9}$ & 4.04 & 20.55 & 19.35 & 19.71 & 13.52 & 23.21 & 19 & -120 \\
OF-P $_{10}$ & 4.09 & 20.39 & 19.64 & 12.73 & 12.90 & 21.92 & 20 & -122 \\
\hline
\end{tabular}


Table S6: Average values for different distances (in Angstroms, Å) and dihedral angles (in degrees) for the nine others "1 ns" trajectories launched for the CF conformer within the polymer matrix.

\begin{tabular}{c|cccccccc}
\hline & $\mathrm{d}_{c c}$ & $\mathrm{~d}_{1}$ & $\mathrm{~d}_{2}$ & $\mathrm{~d}_{3}$ & $\mathrm{~d}_{4}$ & $\mathrm{~d}$ & $\phi_{1}$ & $\phi_{2}$ \\
\hline $\mathbf{C F}_{2}$ & 1.56 & 16.55 & 22.16 & 15.18 & 15.39 & 27.42 & -19 & 5 \\
$\mathbf{C F}_{3}$ & 1.54 & 22.36 & 18.98 & 16.44 & 14.50 & 26.80 & -15 & -2 \\
$\mathbf{C F}_{4}$ & 1.56 & 21.76 & 15.11 & 14.70 & 15.56 & 26.34 & -18 & 5 \\
$\mathbf{C F}_{5}$ & 1.55 & 23.49 & 15.18 & 14.22 & 16.80 & 26.92 & -18 & 0 \\
$\mathbf{C F}_{6}$ & 1.55 & 19.48 & 15.77 & 15.44 & 14.70 & 26.93 & -18 & 4 \\
$\mathbf{C F}_{7}$ & 1.55 & 18.21 & 22.23 & 15.47 & 15.20 & 25.18 & -20 & 5 \\
$\mathbf{C F}_{8}$ & 1.53 & 26.33 & 23.88 & 16.13 & 12.53 & 28.81 & -18 & 1 \\
$\mathbf{C F}_{9}$ & 1.56 & 22.30 & 26.54 & 15.66 & 14.63 & 25.82 & -19 & 6 \\
$\mathbf{C F}_{10}$ & 1.56 & 26.25 & 18.02 & 14.21 & 14.75 & 26.36 & -19 & 4 \\
\hline
\end{tabular}



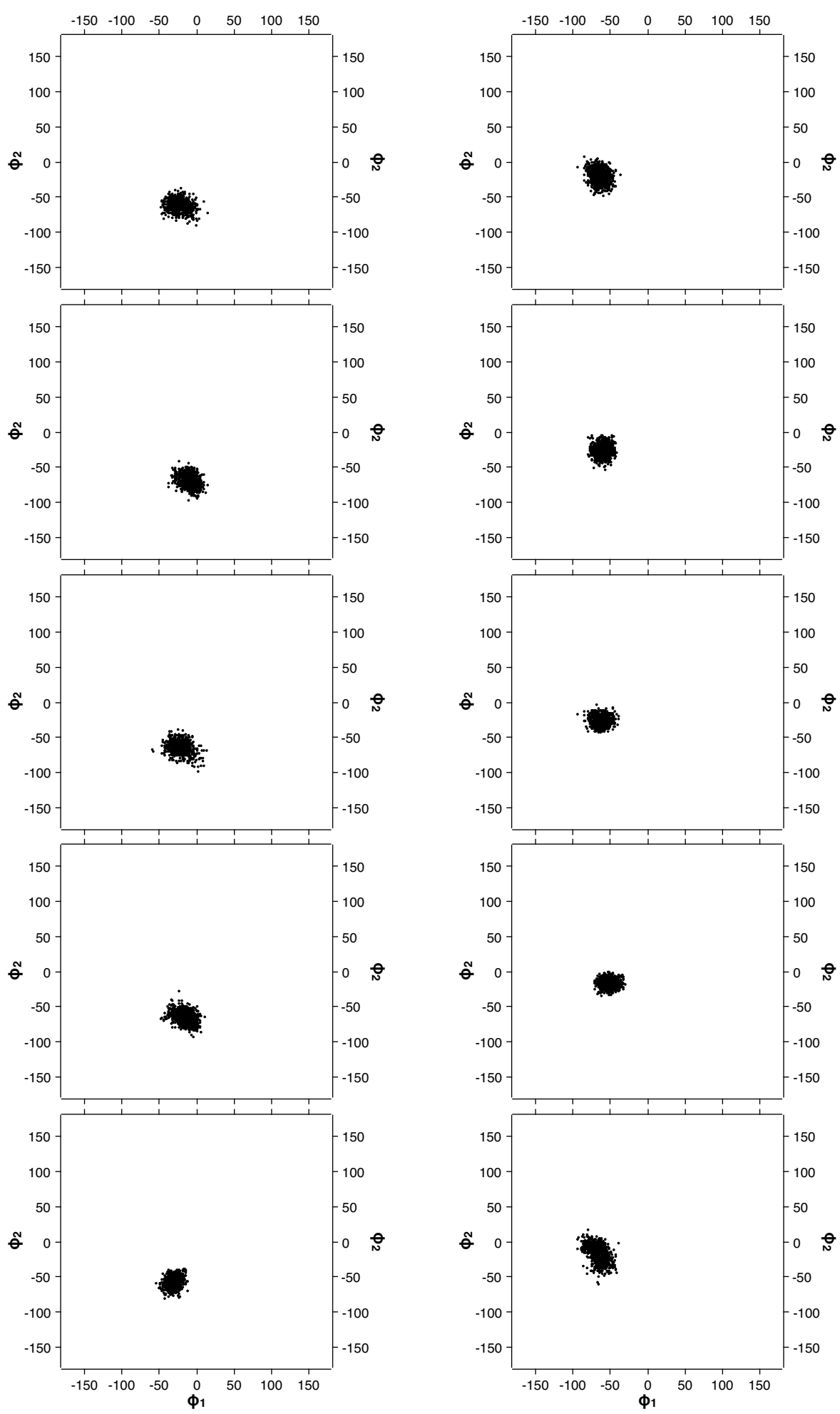

Figure S3: OF-AP isomer embedded in the polymer matrix: representation of value couples $\left(\phi_{1}, \phi_{2}\right)$ (in degrees) for the ten "1 ns " simulations. One graph corresponds to one trajectory. 

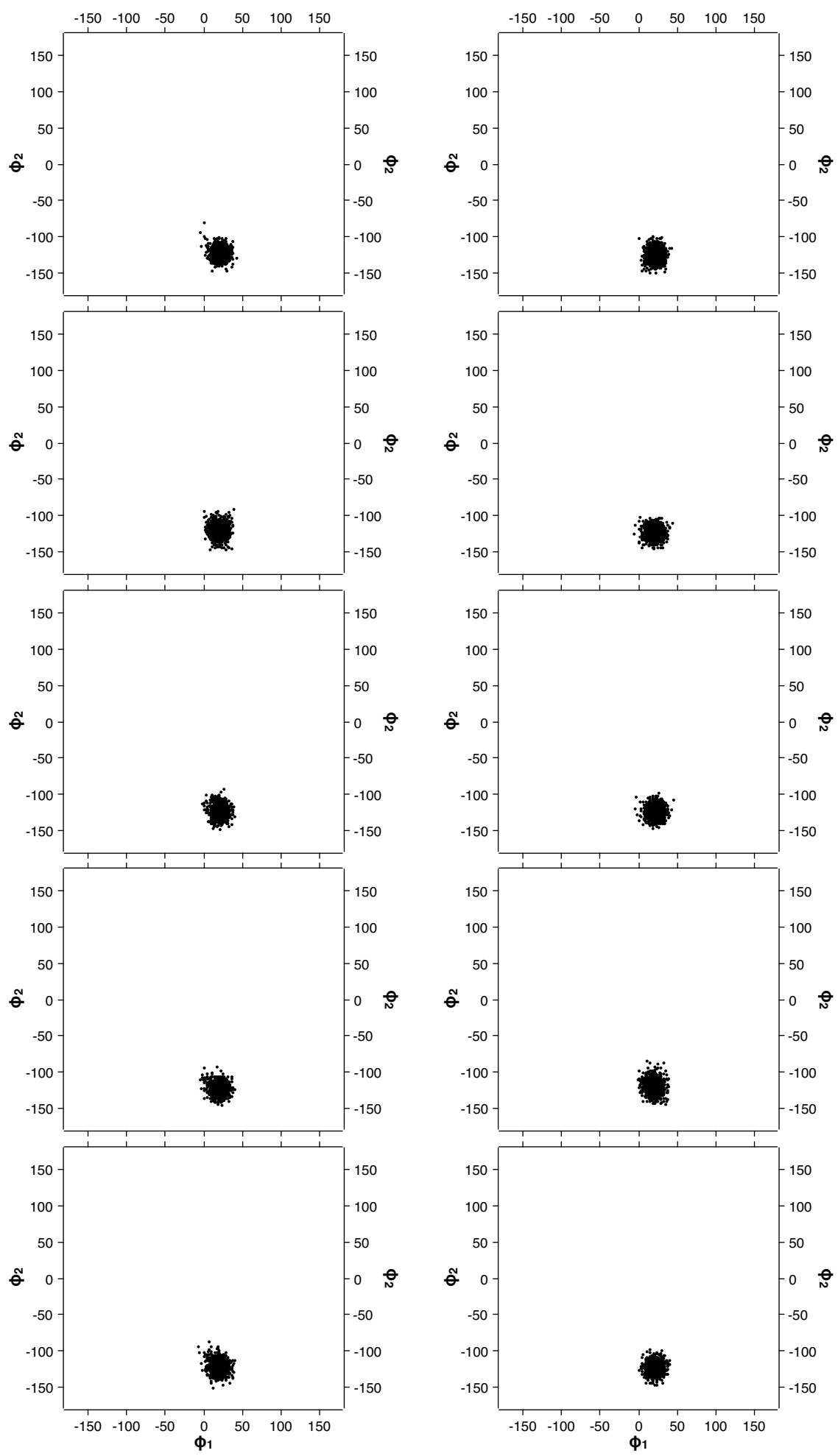

Figure S4: OF-P isomer embedded in the polymer matrix: representation of value couples $\left(\phi_{1}, \phi_{2}\right)$ (in degrees) for the ten "1 ns " simulations. One graph corresponds to one trajectory. 

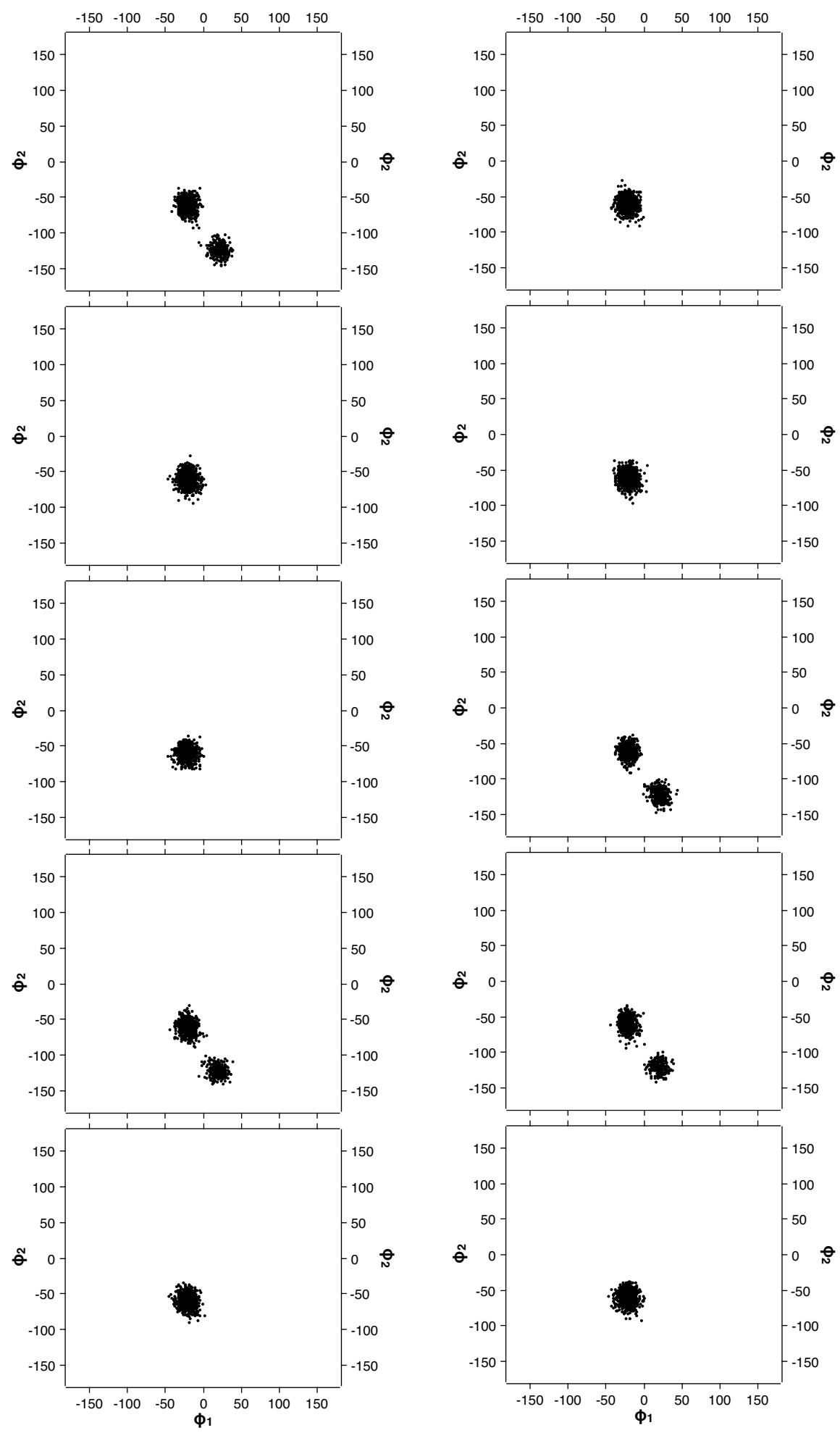

Figure S5: OF-AP isomer in solution (chloroform): representation of value couples $\left(\phi_{1}, \phi_{2}\right)$ (in degrees) for the ten "1 ns "simulations. One graph corresponds to one trajectory. 


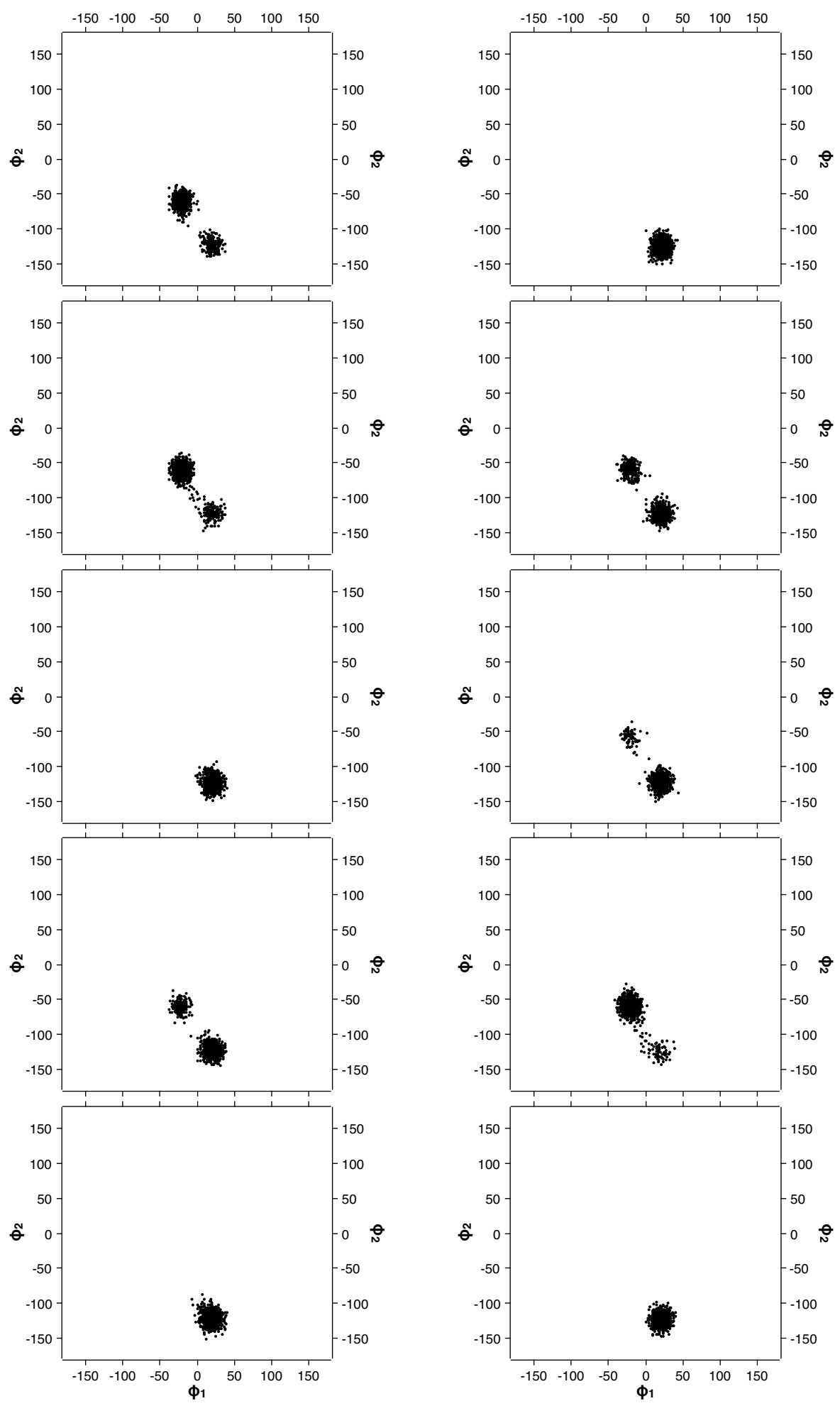

Figure S6: OF-P isomer in solution (chloroform): representation of value couples $\left(\phi_{1}, \phi_{2}\right)$ (in degrees) for the ten "1 ns "simulations. One graph corresponds to one trajectory. 

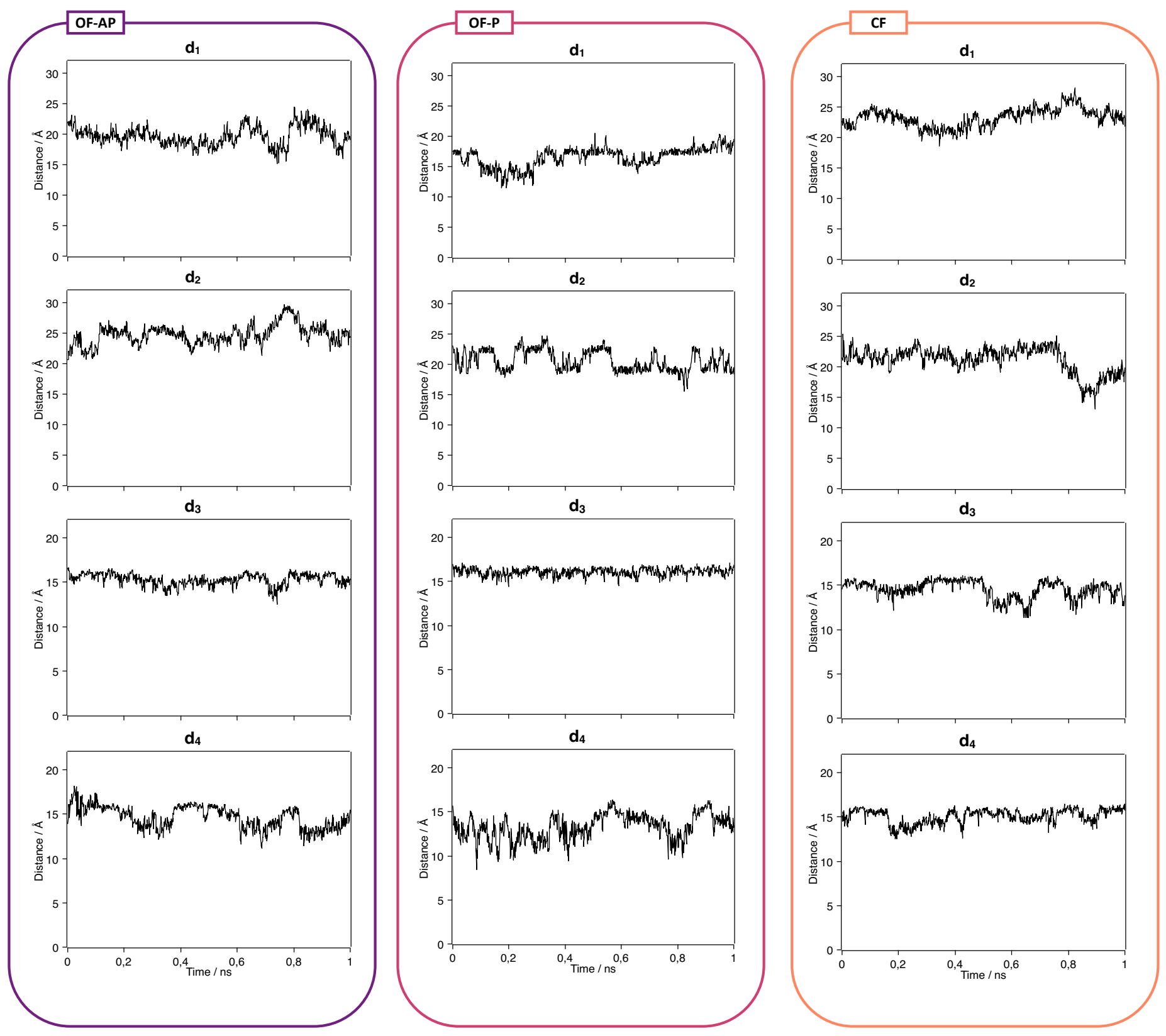

Figure S7: Time evolution of the distances $\mathrm{d}_{i}(\mathrm{i}=1$ to 4$)$ along the selected " 1 ns" trajectory for the $\mathbf{O F}-\mathbf{A P}$ (left), OF-P (middle) and $\mathbf{C F}$ (right) isomers. These parameters are defined on Figure S1. 


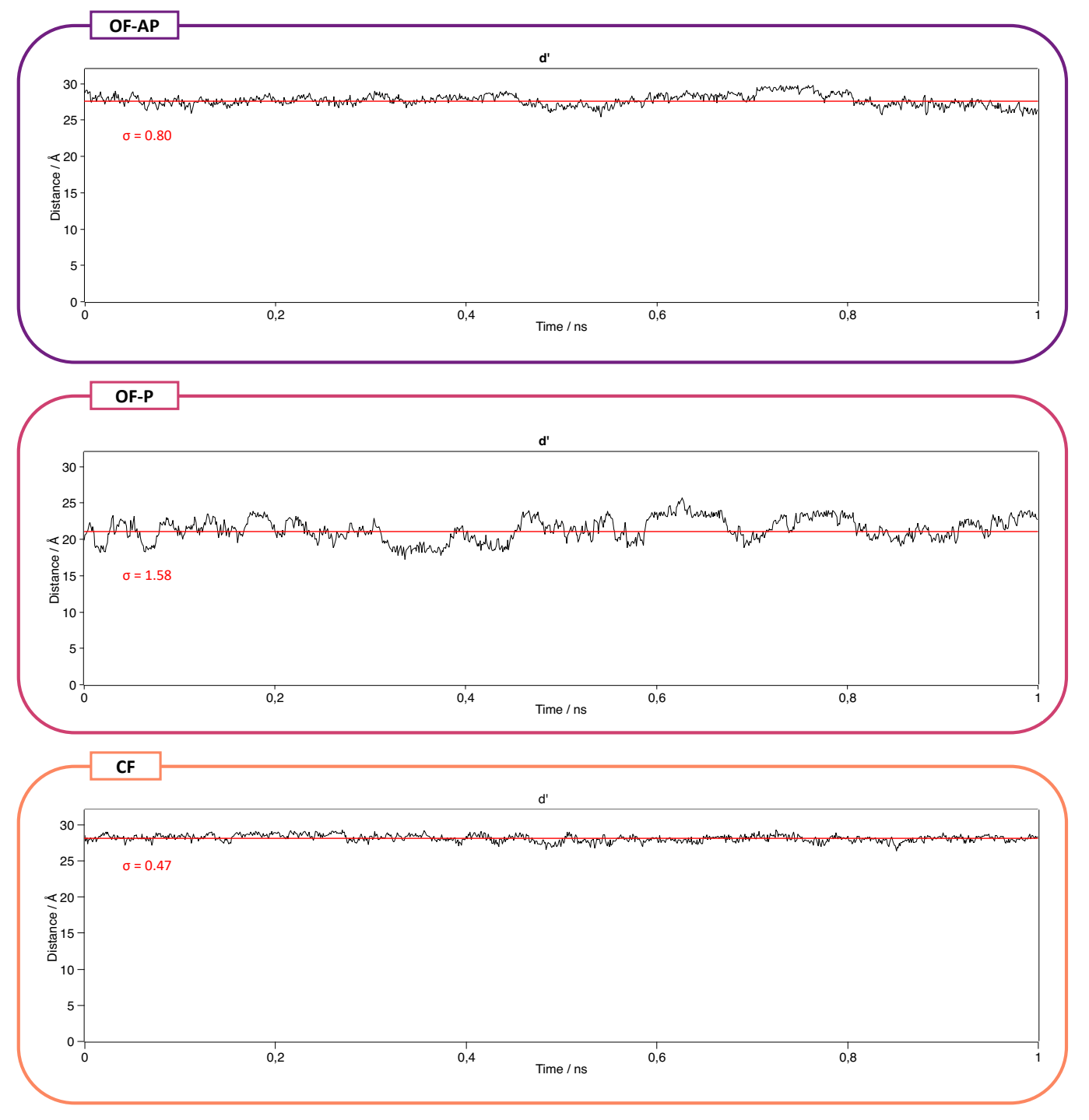

Figure S8: Time evolution of the d distance (in $\AA$ ) along the selected "1 ns" trajectory for the isomers OF-AP (top), OF-P (middle) and CF (bottom).

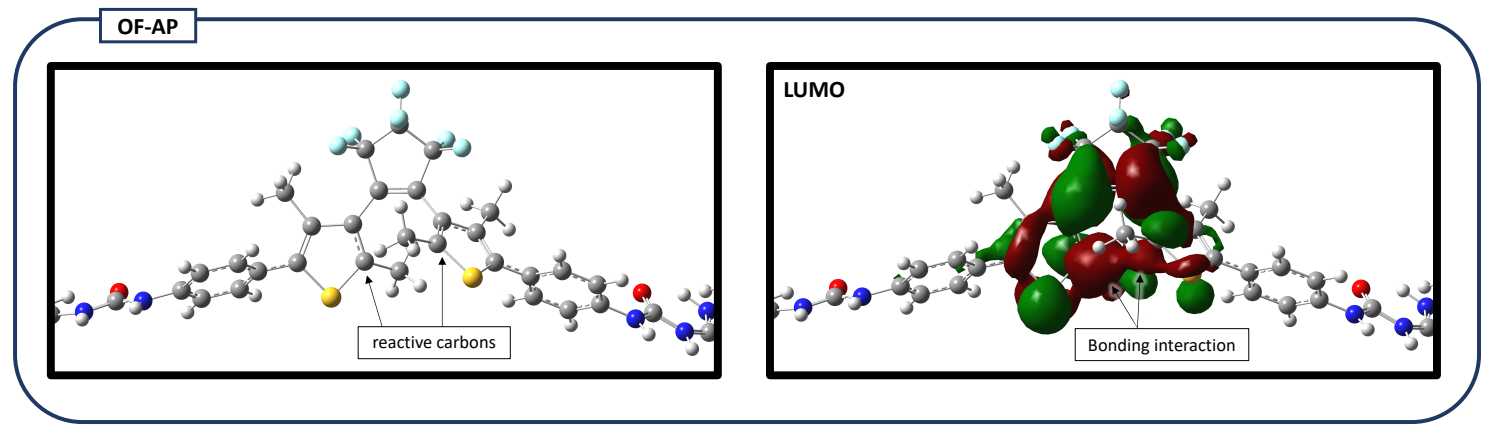

Figure S9: Representation of the LUMO molecular orbital (isodensity=0.025 ua) of $\mathbf{O F}-\mathbf{A P}$. 


\section{Impact of the photoreaction on the polymer matrix: complementary analysis of the MD simulations.}
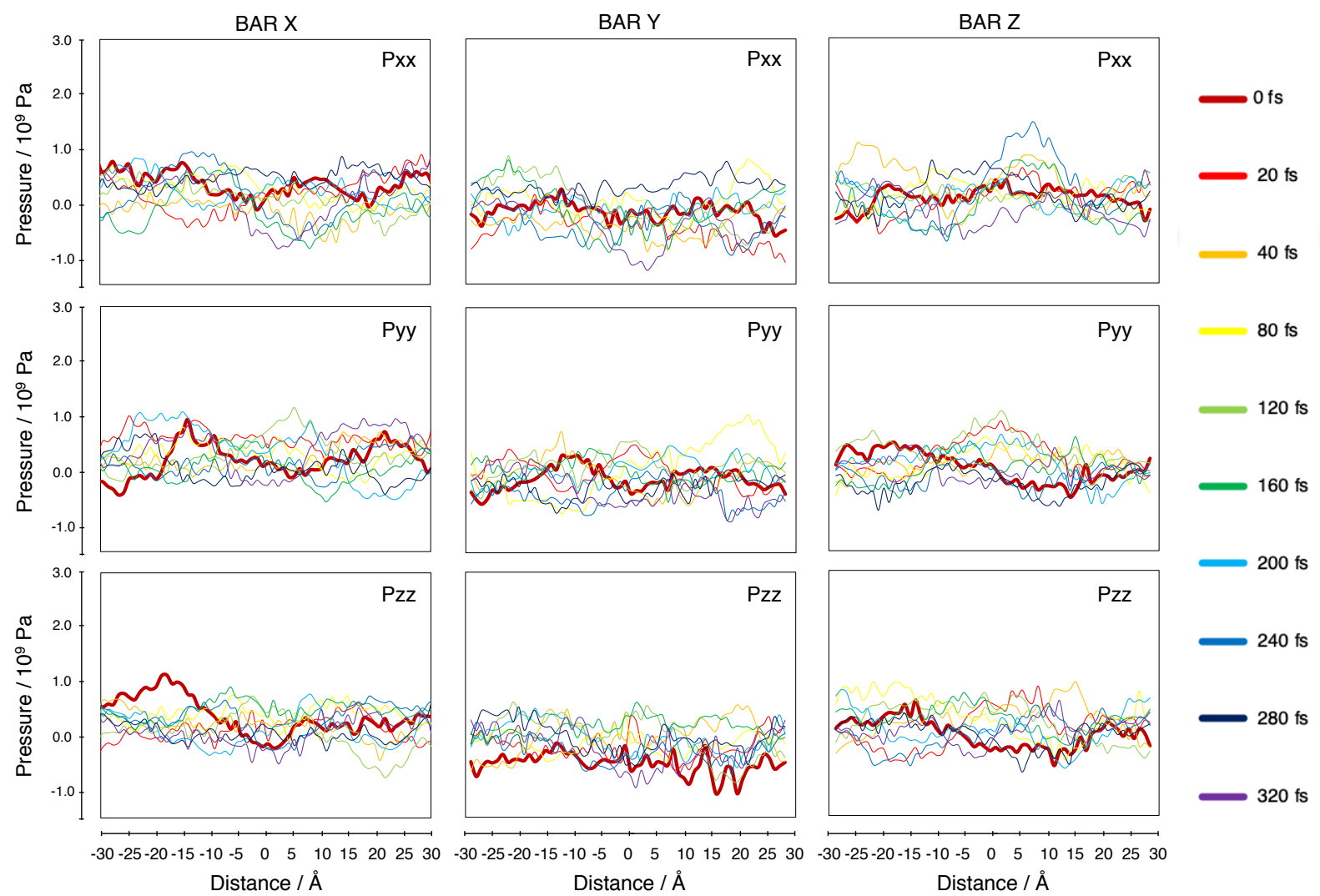

Figure S10: Time evolution of the diagonal components of the pressure tensor $\left(P_{x x}, P_{y y}\right.$ and $\left.P_{z z}\right)$ for each linear section for the ring-closure reaction.

\section{References}

(1) Case, D. et al. AMBER 2018. 2018; University of California, San Francisco. 

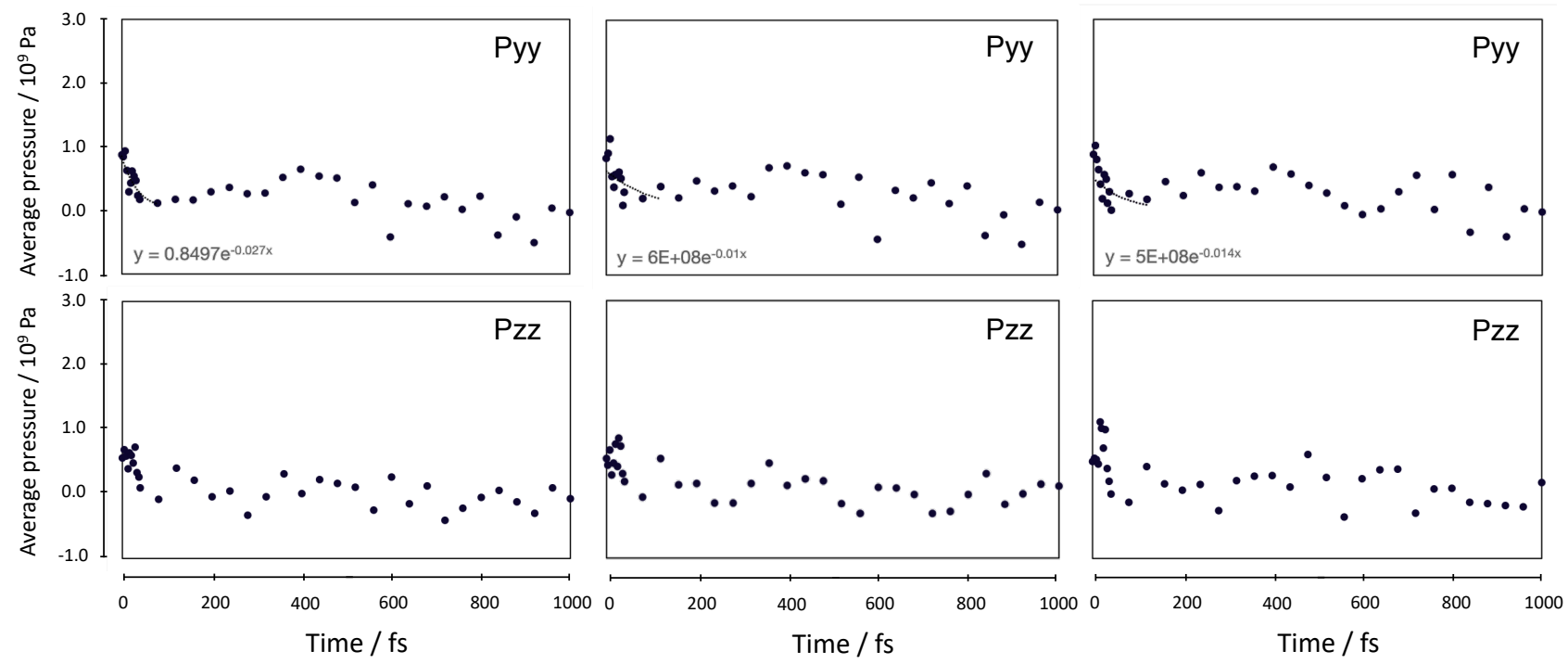

Figure S11: Evolution along the simulation time of the average tensor Pyy (top) and Pzz(bottom) for each bar (X, Y and Z) for the cycloreversion reaction. The average has been done only on the central area (from -5A to 5A) of each bar. This area contains the reactive part of the photochromic molecule and is expected to be the most impacted one. 

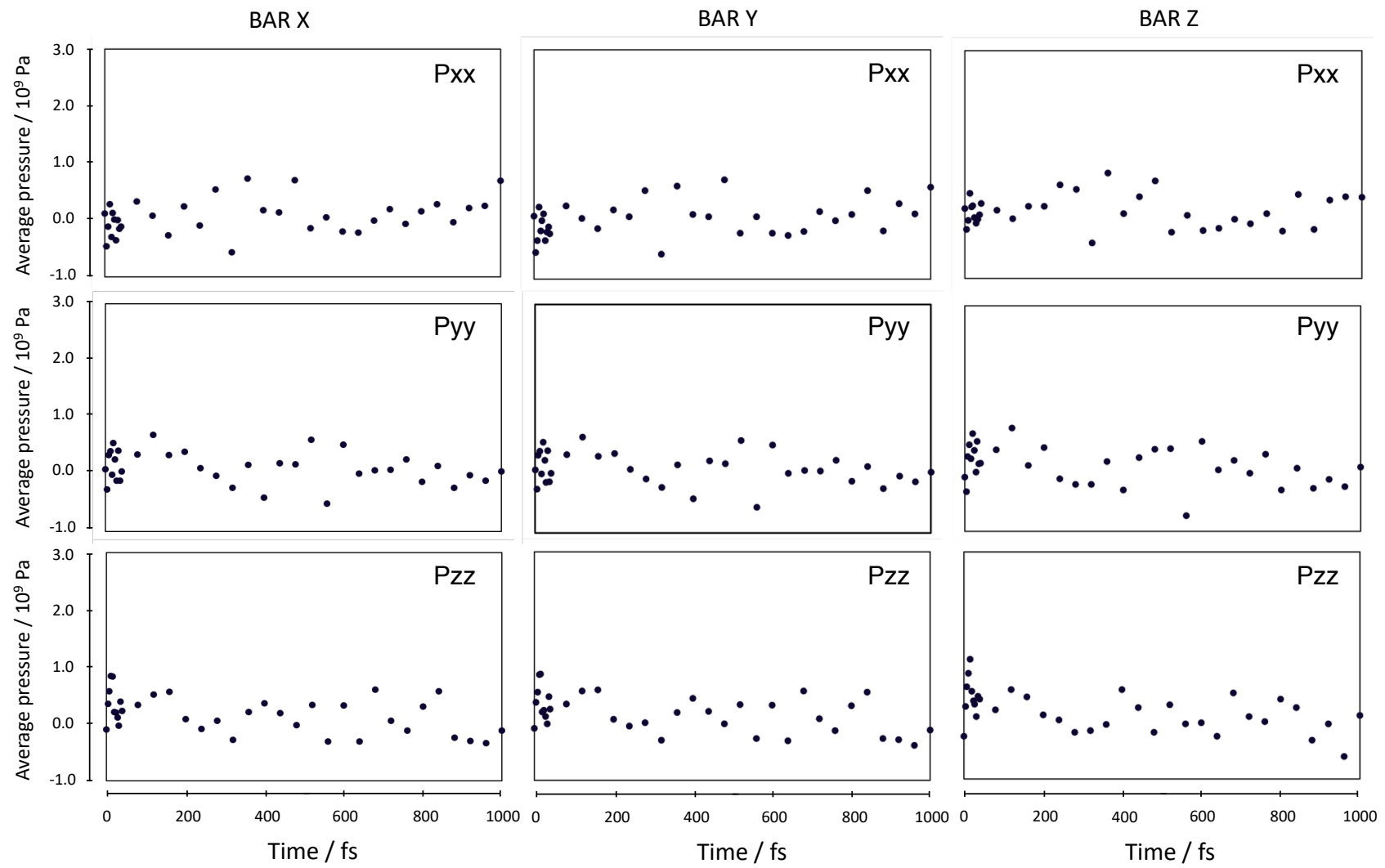

Figure S12: Evolution along the simulation time of the average tensor Pxx (top), Pyy (middle) and Pzz(bottom) for each bar (X, Y and $\mathrm{Z}$ ) for the ring-closure reaction. The average has been done only on the central area (from -5A to 5A) of each bar. This area contains the reactive part of the photochromic molecule and is expected to be the most impacted one. 


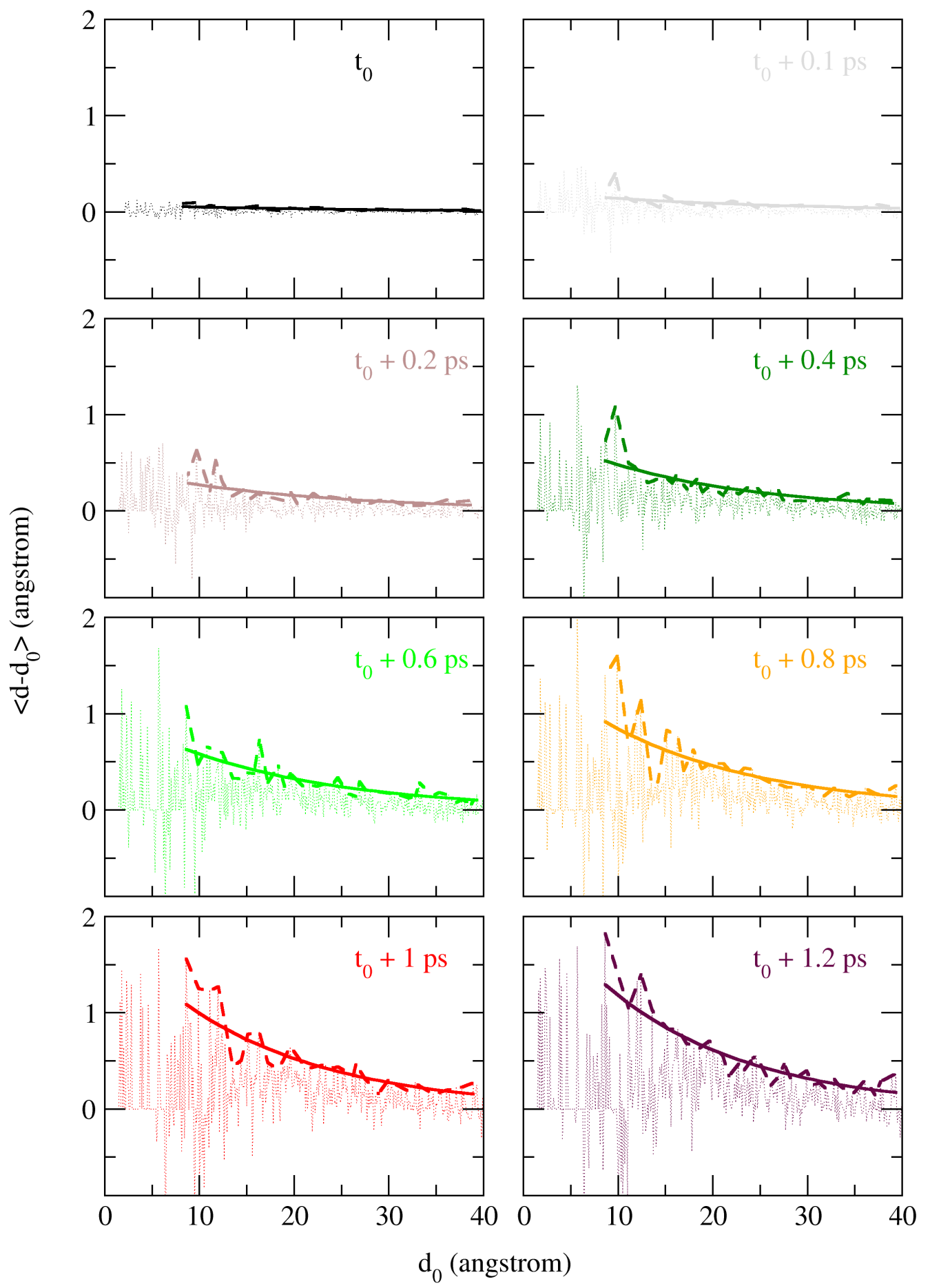

Figure S13: Detail on the computation of the time-dependent distributions of atomic displacements in the polymer matrix after the cycloreversion reaction. For each selected time the raw distribution is the dotted line, the maximum envelop is the bold dashed line, and the fit is the bold full line. The distributions display a large majority of positive displacements (atoms moving away from the photochromic center), but some negative displacements are visible due to complex local rearrangements-16 the polymer. The color code is similar to Figure 12 . 